\title{
DISTRIBUTED CONSENSUS-BASED CALIBRATION OF NETWORKED CONTROL SYSTEMS
}

\author{
$U D C(681.5 .01: 550.831)$
}

\author{
Maja Stankovic ${ }^{1,2}$, Dragan Antić \\ ${ }^{1}$ Telekom Srbija, Beograd, Republic of Serbia \\ ${ }^{2}$ University of Niš, Faculty of Electronic Engineering, Department of Control Systems, \\ Niš, Republic of Serbia
}

\begin{abstract}
In this paper a new algorithm for distributed blind macro-calibration of Networked Control Systems is presented. It is assumed that the measured signal is stochastic and unknown. The algorithm is in the form of recursions of gradient type for estimation of the correction parameters for sensor gains and offsets. The recursion for gain correction is autonomous, derived from the measurement increments. The recursion for offset correction is based on differences between local measurements and utilizes the results of gain correction. It is proved that the algorithm provides asymptotic convergence to consensus in the sense that the corrected gains and offsets are equal for all sensors. It is demonstrated that the adopted structure of the algorithm enables obtaining high convergence rate, superior to the algorithms existing in the literature. Simulation results are provided illustrating the proposed algorithm properties.
\end{abstract}

Key words: networked control systems, blind macro-calibration, sensor, actuator networks, distributed gradient algorithms, consensus

\section{INTRODUCTION}

Lately, great attention has been paid to numerous topics related to Networked Control Systems. A vast literature shows a huge range of deployments within diverse industry fields and robotics, as well as in many multidisciplinary areas, such as surveillance, monitoring, etc. [1,2] New technologies of sensors and actuators, as basic elements of networked control systems, advanced information and communication technologies, as well as powerful distributed algorithms, have driven the entire field to an exceptional development. Continuous development of new applications has led also to new classes of problems, in the domain of both research and development, and engineering applications.

Received May 17, 2019

Corresponding author: Maja Stanković

Telekom Srbija, Takovska 2, Beograd, Republic of Serbia

E-mail: majastanko@gmail.com 
Calibration is still one of very important practical problems for wide deployments of networked control systems and of sensor/actuator networks, in general [3]. Small network control systems and systems in which all sensors are available for adjustments, use the socalled micro-calibration, which consists of calibration of each and every sensor separately. Large networks require specific concepts and methods for calibration, since many sensors could be inaccessible, due to operation in variable and unpredictable conditions. Macrocalibration deals with the calibration problem at the network level [4]. This approach is based on observing the overall networked system response [5,6]. The next step in the development of macro-calibration is the so-called blind macro-calibration. Blind macrocalibration is, in fact, calibration without known stimuli [7-9]. The problem is very complex, conceptually related to the problem of blind deconvolution. The basic idea of blind macrocalibration is that all sensors agree at the network level in such a way that, after calibration, the network behaves as a unified and harmonized sensor/actuator system. The existing approaches start from different a priori assumptions and use different methodologies, but all require centralized actions at the network level [8-10]. The centralized approaches have been found to be neither sufficiently efficient, nor reliable. Recently, improved methods for decentralized blind calibration have arisen as a response to these problems [8]. It is extremely challenging, both conceptually and practically, to develop decentralized algorithms for blind calibration. A methodologically consistent approach to distributed blind calibration has been proposed for the first time in $[7,11]$.

In this paper, an algorithm for distributed blind macro-calibration is proposed. It is based, on one hand, on an analogy between the problems of distributed calibration and distributed time synchronization, and, on the other hand, on recent insights into the basic idea of forming a gradient algorithm for estimation of calibration function parameters in real time $[17,18]$. It is assumed that the measured signal is stochastic and unknown, and that the environment is noiseless. The algorithm results from an attempt to consolidate two methodologies:

1) The methodology of forming gradient schemes starting from local criterion functions of the deviation between the output of the observed sensor and the outputs of its neighbors [7, 12];

2) The methodology of forming recursive procedures for estimation of the parameters in linear calibration functions, suggested within the scope of the time synchronization problem.

Analogies between the problems of sensor calibration and time synchronization can be observed from the basic starting assumptions [13-15].

Namely, in the problem of time synchronization, the basic assumption is that the relative time measured by $i$-th sensor is defined by the relation

$$
\tau_{i}(t)=\alpha_{i} t+\beta_{i}
$$

where $t$ is the absolute time, $\alpha_{i}$ the gain (drift) and $\beta_{i}$ the offset. Ideally, these parameters should be 1 and 0 , respectively. The correction of the sensor output is defined using an affine transformation, which produces the corrected time

$$
T_{i}(t)=a_{i} \tau_{i}(t)+b_{i}=a_{i} \alpha_{i} t+a_{i} \beta_{i}+b_{i}
$$

where $a_{i}$ and $b_{i}$ are parameters which have to be estimated.

In a similar way, in the calibration problem, the output of $i$-th sensor can be represented as

$$
y_{i}(t)=\alpha_{i} x(t)+\beta_{i}
$$


where $\alpha_{i}$ is the gain and $\beta_{i}$ the offset, while $x(t)$ represents the measured signal at the moment $t$. Correction of the sensor output is done by applying the calibration function to the sensor output

$$
z_{i}=a_{i} y_{i}(t)+b_{i}=a_{i} \alpha_{i} x(t)+a_{i} \beta_{i}+b_{i}
$$

where $a_{i}$ and $b_{i}$ are the parameters which have to be estimated.

It is obvious that these two problems are formally similar: the measured signal $x(t)$ in the calibration problem corresponds to the absolute time $t$ in the time synchronization problem; these values are unknown in both cases. However, it should be noted that equating the above mentioned problems is not entirely possible, having in mind different nature of the functions $x(t)$ and $t$, as well as the essence of the technical setting of the corresponding problems. As a consequence, these two problems are treated in the literature in different, methodologically autonomous ways.

In one of important papers in the area of time synchronization in sensor networks, the authors deal with the problem starting methodologically from the idea of consensus [14]. Estimation of gain and offset parameters is done by recursions which generate estimates of these two parameters in real time. The authors have proved that it is possible in the deterministic case to achieve consensus on local times for all the sensors in a network. Estimation of gain correction parameters is done first by a recursive scheme which is based on the increments of the input function $t$ (which does not depend on offset). In such a way, all sensors in the network asymptotically achieve identical equivalent gains. After this step, after applying the previously estimated gain parameter, the offset is estimated by a separate recursion, which leads to the complete equalization of the local times of all the sensors in the network. Recently, a family of time synchronization algorithms based on consensus (CBTS - Consensus Based Time Synchronization) has been presented in a unified way in $[16,17]$.

The main idea of this paper is to construct a novel distributed blind macro calibration algorithm using as the structure of the CBTS algorithms as a starting point. The calibration algorithm which will be proposed in this paper consists of:

1) Independent recursive gradient algorithm for estimation of the sensor gain correction parameters, based on the model of increments of the sensor output signal;

2) Recursive gradient algorithm for estimation of the sensor offset correction parameters, which is based on the current results taken from the algorithm for estimation of the gain correction parameter.

It is to be emphasized that the proposed algorithm for gain correction parameter estimation differs structurally from the analogous algorithms from the CBTS family: it is much simpler and. moreover, linear-in-the-parameters. A particular quality of the algorithm in the sense of practical engineering applications lies in its computational and communicational simplicity. Having in mind the separated estimation of the gain correction parameters, it is to be expected that the corresponding convergence rate is superior to the one obtainable with the algorithm which estimates both correction parameters together by a higher order gradient scheme [7, $11,18]$

In the theoretical part, proofs are provided for convergence of the proposed recursions to consensus in the mean square sense. In the experimental part, it is demonstrated by simulations that algorithm outperforms all similar algorithms from the literature in the sense of convergence rate; this is of an extreme importance for practice. 
Formally, the paper is organized as follows. Section 2 deals with the basic setting for the proposed algorithms. Section 3 is devoted to the offset correction parameter, assuming that the sensor gain is equal to one for all sensors in the network. In this way the essence of the algorithm is clearly explained. In Section 4, the algorithm for gain correction estimation is presented, while section 5 deals with simultaneous estimation of both the corrected gain and the corrected offset. All the convergence proofs are presented in extenso. Section 6 is devoted to selected simulation results.

\section{BASIC SETTING}

We consider $\mathrm{n}$ distributed sensors measuring a discrete-time stochastic signal $x(t)$. We assume that the output of $i$-th sensor is represented by

$$
y_{i}(t)=\alpha_{i} x(t)+\beta_{i}
$$

where $\alpha_{i}$ and $\beta_{i}$ are, in general, the unknown sensor gain and offset, respectively. By sensor calibration we consider the application of an affine calibration transformation, which produces the corrected sensor output

$$
z_{i}(t)=a_{i} y_{i}(t)+b_{i}=g_{i} x(t)+f_{i}
$$

where $a_{i}$ and $b_{i}$ are the calibration parameters, while $g_{i}=a_{i} \alpha_{i}$ and $f_{i}=a_{i} \beta_{i}+b_{i}$ represent the corrected gain and the corrected offset, respectively. Ideally, the role of the parameters $a_{i}$ and $b_{i}$ is to compensate the influence of unknown parameters $\alpha_{i}$ and $\beta_{i}$ in such a way as to obtain $g_{i}$ close to one and $f_{i}$ close to zero, $i=1, \ldots, n$.

Assume that the observed sensors form a network with a specific structure, which can formally be represented by a directed graph $G(N, L)$, where $N$ is the set of nodes and $L$ the set of arcs. The adjacency matrix $A=\left[a_{i j}\right], i, j=1, \ldots, n$, is such that $a_{i j}=1$ if the $\mathrm{j}$-th sensor can send its message to the $\mathrm{i}$-th sensor, and $a_{i j}=0$ otherwise; the corresponding arc is directed from $\mathrm{j}$ to i. Let $N_{i}$ be the set of neighboring nodes of the i-th node, i.e., the set of nodes $\mathrm{j}$ for which $a_{i j}=1$. The aim of the algorithm for distributed blind macro-calibration is to estimate the calibration parameters $a_{i}$ and $b_{i}$ in a distributed manner and in real-time, without the explicit knowledge of the measured signal.

\section{ESTIMATION OF THE OFFSET CALIBRATION PARAMETER}

We shall first concentrate on the problem of offset correction $\left(\alpha_{i}=1\right.$ in (5)). The algorithm for estimation of $b_{i}$, without any pretension to offer a complete offset compensation for all sensors, should enable, through a global consensus mechanism, a dominant influence of well calibrated sensors with respect to those that are not.

Assume that $x(t)$ is a discrete time stochastic process. We introduce, like in [7] and [12], the following set of local criteria

$$
J_{i}=\sum_{j \in N_{i}} \gamma_{i j} E\left\{\left(z_{j}(t)-z_{i}(t)\right)^{2}\right\}
$$

$i=1,2, \ldots, n$, where $\gamma_{i j}>0, j \in N_{i}$, are a priori chosen scalar weights which represent the relative importance of the in-neighboring nodes. The gradient of (7) is given by 


$$
\operatorname{grad}_{b_{i}} J_{i}=\frac{\partial J_{i}}{\partial b_{i}}=-2 \sum_{j \in N_{i}} \gamma_{i j} E\left\{\left(z_{j}(t)-z_{i}(t)\right)\right\}
$$

From here, it directly follows that a gradient recursion for estimating parameter $b_{i}$ is given by

$$
\hat{b}_{i}(t+1)=\hat{b}_{i}(t)+\delta_{i}(t) \sum_{j \in N_{i}} \gamma_{i j} \varepsilon_{i j}(t)
$$

where $\hat{b}_{i}(t)$ is an estimate of parameter $b_{i}$ at the moment $\mathrm{t}, \delta_{i}(t)>0$ is the gain of the algorithm (step size) which affects its convergence rate, while $\varepsilon_{i j}(t)=\hat{z}_{j}(t)-\hat{z}_{i}(t)$, where $\hat{z}_{i}(t)=y_{i}(t)+\hat{b}_{i}(t)$. The initial condition $\hat{b}_{i}(0)$, determined as an a priori information of sensor characteristics, is set, in general, to $\hat{b}_{i}(0)=0, i=1,2, \ldots, n$. Notice that the recursion (9) subsumes availability of local current corrected sensor outputs communicated only by the neighboring nodes, $j \in N_{i}$. The underlying idea is to achieve $\hat{z}_{j}(t)=\hat{z}_{i}(t), i, j=1,2, \ldots, n$, by minimizing all the local criteria, so that all the estimates $\hat{f}_{i}(t)=\beta_{i}+\hat{b}_{i}(t)$ tend asymptotically to the same value. In this respect, it is convenient to transform relation (9) in the following way

$$
\hat{f}_{i}(t+1)=\hat{f}_{i}(t)+\delta_{i}(t) \sum_{j \in N_{i}} \gamma_{i j}\left(\hat{f}_{j}(t)-\hat{f}_{i}(t)\right) \text {. }
$$

All the recursions from (10) can be represented for all the nodes in the network in a compact vector-matrix form

where

$$
\hat{f}(t+1)=[\mathrm{I}+\Delta(t) \Gamma] \hat{f}(t)
$$

and

$$
\begin{gathered}
\hat{f}(t)=\left[\hat{f}_{1}(t) \ldots \hat{f}_{n}(t)\right]^{T} \\
\Delta(t)=\operatorname{diag}\left\{\delta_{1}(t), \ldots, \delta_{n}(t)\right\}
\end{gathered}
$$

$$
\Gamma=\left[\begin{array}{cccc}
-\sum_{\mathrm{j}} \gamma_{1 \mathrm{j}} & \gamma_{12} & \cdots & \gamma_{1 \mathrm{n}} \\
\gamma_{21} & -\sum_{\mathrm{j}} \gamma_{2 \mathrm{j}} & \cdots & \gamma_{2 \mathrm{n}} \\
\vdots & \vdots & \ddots & \vdots \\
\gamma_{\mathrm{n} 1} & \gamma_{\mathrm{n} 2} & \cdots & -\sum_{\mathrm{j}} \gamma_{\mathrm{nj}}
\end{array}\right]
$$

represents a weighted Laplacian of the graph $G(N, L)$.

Recursion (11) represents a linear dynamic system with variable parameters $\Delta(t)$. It can be analyzed, in general, using the methodology of analysis of dynamic discrete consensus schemes. Matrix $\Gamma$ plays the key role in this analysis; however, the elements of this matrix are not real communication gains within the network, but they represent a priori determined weights introduced above by the very definition of criteria $J$ (see also the related comments below). It should also be emphasized that the communication delay is considered to be negligible w.r.t. bandwidth of the measured signal, having in mind typical real situations in which the network is implemented using a high speed wireless sensor network and the measurements are connected to slow processes, like temperature, pressure, humidity, etc.

In the basic setting, we assume:

A) $\delta_{i}(t)=\delta=$ const

B) Graph $G$ has a center node (i.e. a node from which all the other nodes are reachable). 
Assumption A) is typical for gradient schemes in the noiseless case (measurement and communication noises are absent). Assumption B) is very common for various problems related to dynamic consensus. Intuitively, it means that there is at least one node in the network which can communicate with all the other nodes. In such a way, isolation of some nodes, which could inhibit the achievement of consensus, is effectively prevented. Formal consequences of this assumption are discussed in many papers [6,19-21].

Lemma 1: Let Assumption B) be satisfied. Then matrix $\Gamma$ has one simple eigenvalue at the origin and the remaining ones have negative real parts.

Lemma 1 is of key importance for the whole analysis. The proof of Lemma 1 can be found in [6, 20-22].

Define vector $\mathbf{1}=\left[\begin{array}{lll}1 & \cdots & 1\end{array}\right]^{T}$. According to [20], this vector represents the right eigenvector of $\Gamma$ corresponding to the zero eigenvalue. Let $\pi$ be the corresponding left eigenvector, satisfying $\pi \Gamma=0$ and $\pi \mathbf{1}=1$. According to Lemma 1 and the condition $\pi \mathbf{1}=1$, this eigenvector is unique.

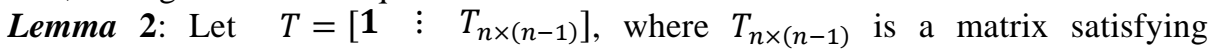
$\operatorname{spanT}_{n \times(n-1)}=\operatorname{span} \Gamma$. Then $T$ is a non-singular matrix and

$$
T^{-1} \Gamma T=\left[\begin{array}{ccc}
0 & \vdots & 0_{1 \times(n-1)} \\
0_{(n-1) \times 1} & \vdots & \Gamma^{*}
\end{array}\right] .
$$

where (n-1) $x(n-1)$ matrix $\Gamma^{*}$ is Hurwitz.

The proof of Lemma 2 is directly based on the Jordan's form of matrix $\Gamma[7,12]$.

Theorem 1: Let Assumptions A) and B) be satisfied. Then, there exists a scalar $\delta^{\prime}>0$ such that for all $0<\delta<\delta^{\prime}$ in (11)

$$
\lim _{t \rightarrow \infty} \hat{f}(t)=f_{\infty}=1 \pi \hat{f}(0)
$$

where $\hat{f}(0)=\left[\begin{array}{lll}\beta_{1} & \ldots & \beta_{n}\end{array}\right]^{T}$.

Proof: We define $\tilde{f}(t)=T^{-1} \hat{f}(t)$. Then, from (11) we obtain

$$
\tilde{f}(t+1)=\left(I+\delta T^{-1} \Gamma T\right) \tilde{f}(t) .
$$

According to (13), if $\tilde{f}(t)=\left[\tilde{f}(t)^{[1] T} \quad \tilde{f}(t)^{[2] T}\right]^{T}$, we obtain directly

$$
\begin{gathered}
\tilde{f}(t+1)^{[1]}=\tilde{f}(t)^{[1]} \\
\tilde{f}(t+1)^{[2]}=\left(\mathrm{I}+\delta \Gamma^{*}\right) \tilde{f}(t)^{[2]}
\end{gathered}
$$

where $\operatorname{dim} \tilde{f}(t)^{[1]}=1$ and $\operatorname{dim} \tilde{f}(t)^{[2]}=n-1$. Having in mind that matrix $\Gamma$ has one zero eigenvalue and the remaining ones with negative real parts, it follows from Lemma 2 that matrix $\Gamma^{*}$ is Hurwitz, i.e., all its eigenvalues have strictly negative real parts. Consequently, there exists such $\delta^{\prime}>0$ that for all $0<\delta<\delta^{\prime}$ the condition $\max _{i}\left|\lambda_{i}(I+\delta \Gamma)\right|<1$ is fulfilled. This implies that $\lim _{t \rightarrow \infty} \tilde{f}(t)^{[2]}=0$, so that we get

$$
\lim _{t \rightarrow \infty} \tilde{f}(t)=\left[\begin{array}{llll}
\tilde{f}(0)^{[1]} & 0 & \cdots & 0
\end{array}\right]^{T}
$$

i.e. $f_{\infty}=T\left[\tilde{f}(0)^{[1]} \quad 0 \quad \cdots \quad 0\right]^{T}=\mathbf{1} \pi \hat{f}(0)$.

According to the given proof, it is clear that the algorithm (11) achieves the asymptotic consensus in such a way that the equivalent offsets for all the nodes in the 
network become equal. The speed of achieving this condition is exponential. From Theorem 1 we can conclude that concrete values of the common equivalent offset depend on the unknown sensor offsets and the adopted weighting coefficient values $\gamma_{i j}$ from (7).

\section{ESTIMATION OF THE GAIN CORRECTION PARAMETER}

Starting from (3), we define $\Delta y_{i}(t)=y_{i}(t+1)-y_{i}(t)$ and $\Delta x(t)=x(t+1)-$ $x(t)$, and get for the increment of the output of $i$-th sensor

$$
\Delta y_{i}(t)=\alpha_{i} \Delta x(t)
$$

If, in general, the sensor calibration function is given by (6), we get, after correction, that

$$
z_{i}(t)=a_{i} \alpha_{i} x(t)+a_{i} \beta_{i}+b_{i}
$$

where $g_{i}=a_{i} \alpha_{i}$ and $f_{i}=a_{i} \beta_{i}+b_{i}$.

The aim of the gain correction algorithm is to estimate parameter $a_{i}$ in such a way that all the sensors in the network have asymptotically the same equivalent gains $g_{i}=a_{i} \alpha_{i}$. The algorithm will be derived starting from the relation

$$
\Delta z_{i}(t)=a_{i} \alpha_{i} \Delta x(t)=a_{i} \Delta y_{i}(t)
$$

using the previously explained methodology in 3 . Thus, we define a set of local criteria

$$
J_{i}^{\Delta}=\sum_{j \in N_{i}} \gamma_{i j} E\left\{\left(\Delta z_{j}(t)-\Delta z_{i}(t)\right)^{2}\right\}
$$

and

$$
\frac{\partial J_{i}^{\Delta}}{\partial a_{i}}=-\sum_{j \in N_{i}} \gamma_{i j} E\left\{\left(\Delta z_{j}(t)-\Delta z_{i}(t)\right) \Delta y_{i}(t)\right\}
$$

From here we get a gradient recursion for estimating parameter $a_{i}$

$$
\hat{a}_{i}(t+1)=\hat{a}_{i}(t)+\delta_{i}(t) \sum_{j \in N_{i}} \gamma_{i j} \varepsilon_{i j}^{\Delta}(t) \Delta y_{i}(t)
$$

where $\hat{a}_{i}(t)$ is an estimate of the parameter $a_{i}$ at the moment $\mathrm{t}, \delta_{i}(t)>0$ is the step size of the algorithm, while $\varepsilon_{i j}^{\Delta}(t)=\Delta \hat{z}_{j}(t)-\Delta \hat{z}_{i}(t)$, where $\Delta \hat{z}_{i}(t)=\hat{a}_{i}(t) \Delta y_{i}(t)$. The relation (26) can be represented in terms of the corrected gains in the following way

$$
\hat{g}_{i}(t+1)=\hat{g}_{i}(t)+\delta_{i}(t) \sum_{j \in N_{i}} \gamma_{i j}\left(\hat{g}_{j}(t)-\hat{g}_{i}(t)\right) \alpha_{i}^{2} \Delta x(t)^{2}
$$

All the recursions from (27) can be represented in a compact vector-matrix form as

$$
\hat{g}(t+1)=\left[\mathrm{I}+\Delta x(t)^{2} \Delta(t) \alpha^{2} \Gamma\right] \hat{g}(t)
$$

where $\hat{g}(t)=\left[\begin{array}{lll}\hat{g}_{1}(t) & \cdots & \hat{g}_{n}(t)\end{array}\right]^{T}$ and $\alpha=\operatorname{diag}\left\{\alpha_{1}, \ldots, \alpha_{n}\right\}$.

Relation (28) is similar to relation (11); the main difference lies is in the presence of the stochastic element $\Delta x(t)^{2}$ (which is not measurable). Therefore, the related methodology of convergence analysis becomes more complicated than the one for the offset correction algorithm. 
For the purpose of convergence analysis, we assume

$$
\text { C) } \begin{aligned}
E & \left.E \Delta x(t)^{2} \mid F_{t}\right\} \geq \sigma_{c}^{2}>0 \\
E & \left.E \Delta x(t)^{4} \mid F_{t}\right\} \leq \sigma_{c}^{4}<\infty
\end{aligned}
$$

where $F_{t}$ is the minimal $\sigma$-algebra generated by $\{x(t), x(t-1), \ldots\}$. Intuitively, the meaning of this assumption is the following: the signal does not have a trivial constant value and has the bounded fourth moment; in practice, both conditions are easily fulfilled. In general, the assumption about stochastic nature of the signal arises naturally from the fact that its samples are unknown for the observer implementing the proposed calibration algorithm function. The assumption $\mathrm{C}$ ) is very mild, easily achievable in practice.

Theorem 2: Let Assumptions A), B) and C) be satisfied. Then there exists $\delta^{\prime \prime}>0$ such that for all $\delta<\delta^{\prime \prime \prime}$ in (28) $\hat{g}(t)$ tends exponentially to consensus in the mean square sense.

Proof: Let $\mathrm{T}_{1}$ be the transformation matrix which has the same role as the transformation $\mathrm{T}$ in Lemma 2, but now with respect to the matrix $\alpha^{2} \Gamma$, which has, also, one eigenvalue at the origin and the remaining ones with negative real parts. If we assume $\tilde{g}(t)=\mathrm{T}_{1}^{-1} \hat{g}(t)$, we get from (28) that

$$
\tilde{g}(t+1)=\left(\mathrm{I}+\delta \mathrm{T}_{1}^{-1} \alpha^{2} \Gamma \mathrm{T}_{1} \Delta x(t)^{2}\right) \tilde{g}(t)
$$

According to Lemma 2, we obtain

$$
\begin{gathered}
\tilde{g}(t+1)^{[1]}=\tilde{g}(t)^{[1]} \\
\tilde{g}(t+1)^{[2]}=\left(\mathrm{I}+\delta \Delta x(t)^{2} \Gamma_{\alpha}^{*}\right) \tilde{g}(t)^{[2]}
\end{gathered}
$$

where $\Gamma_{\alpha}^{*}$ follows from the relation

$$
\mathrm{T}_{1}^{-1} \alpha^{2} \Gamma \mathrm{T}_{1}=\left[\begin{array}{cc}
0 & 0 \\
0 & \Gamma_{\alpha}^{*}
\end{array}\right]
$$

Like $\Gamma^{*}$ in Lemma 2, all the eigenvalues of $\Gamma_{\alpha}^{*}$ have all the eigenvalues with negative real parts. Consequently, there exists such a symmetric positive definite matrix $R_{\alpha}^{*}$ that satisfies the Lyapunov equation

$$
\Gamma_{\alpha}^{* T} R_{\alpha}^{*}+R_{\alpha}^{*} \Gamma_{\alpha}^{*}=-Q_{\alpha}^{*}
$$

where $Q_{\alpha}^{*}$ is a symmetric positive definite matrix. Further, we define $v(t)=$ $E\left\{\tilde{g}(t)^{[2] T} R_{\alpha}^{*} \tilde{g}(t)^{[2]}\right\}$. From (31) we obtain

$$
v(t+1)=E\left\{\tilde{g}(t)^{[2] T}\left[\begin{array}{c}
I+\delta E\left\{\Delta x(t)^{2} \mid F_{t}\right\}\left(\Gamma_{\alpha}^{* T} R_{\alpha}^{*}+R_{\alpha}^{*} \Gamma_{\alpha}^{*}\right)+ \\
\delta^{2} E\left\{\Delta x(t)^{4} \mid F_{t}\right\} \Gamma_{\alpha}^{* T} R_{\alpha}^{*} \Gamma_{\alpha}^{*}
\end{array}\right] \tilde{g}(t)^{[2]}\right\} .
$$

We shall first analyze the elements of the right side of (34). According to (33), for the linear part we have

$$
-E\left\{\tilde{g}(t)^{[2] T} \delta E\left\{\Delta x(t)^{2} \mid F_{t}\right\} Q_{\alpha}^{*} \tilde{g}(t)^{[2]}\right\} \leq-\delta \sigma_{c}^{2} \min _{i} \lambda_{i}\left(Q_{\alpha}^{*}\right) E\left\{\tilde{g}(t)^{[2] T} \tilde{g}(t)^{[2]}\right\}
$$

For the quadratic part, we have the upper bound

$$
\delta^{2} \sigma_{c}^{4} E\left\{\tilde{g}(t)^{[2] T} \Gamma_{\alpha}^{* T} R_{\alpha}^{*} \Gamma_{\alpha}^{*} \tilde{g}(t)^{[2]}\right\} .
$$


If we take into account that for a given $\mathrm{n}$-dimension vector $x$

$$
x^{T} Q_{\alpha}^{*} x \geq \lambda_{\min }\left(Q_{\alpha}^{*}\right) x^{T} x=\lambda_{\min }\left(Q_{\alpha}^{*}\right) x^{T} R_{\alpha}^{* \frac{1}{2}} R_{\alpha}^{*-1} R_{\alpha}^{* \frac{1}{2}} x \geq \lambda_{\min }\left(Q_{\alpha}^{*}\right) \frac{1}{\max _{i} \lambda_{i}\left(R_{\alpha}^{*}\right)} x^{T} R_{\alpha}^{*} x
$$

and also

$$
x^{T} \Gamma_{\alpha}^{* T} R_{\alpha}^{*} \Gamma_{\alpha}^{*} x \leq \max _{i} \lambda_{i}\left(\mathrm{R}_{\alpha}^{*}\right) \max _{i} \lambda_{i}\left(\Gamma_{\alpha}^{* T} \Gamma_{\alpha}^{*}\right) x^{T} x
$$

where $x^{T} x \leq \frac{v(t)}{\min _{i} \lambda_{i}\left(\mathrm{R}_{\alpha}^{*}\right)}$, we get

$$
v(t+1) \leq\left(1-\delta k_{1}^{*}+\delta^{2} k_{2}^{*}\right) v(t)
$$

where $k_{1}^{*}>0$ and $k_{2}^{*}>0$ are generic constants. Since the minimum of the quadratic parabola $y=1-k_{1}^{*} x+k_{2}^{*} x^{2}$ is at $x_{\min }=\frac{k_{1}^{*}}{2 k_{2}^{*}}>0$, it follows that there exists such $\delta^{\prime \prime \prime}>0$ that $0<1-k_{1}^{*} \delta+k_{2}^{*} \delta^{2}<1$ for all $0<\delta<\delta^{\prime \prime \prime}$. From this, we obtain that $v(t) \rightarrow 0$ exponentially when $t \rightarrow \infty$, i.e, $\tilde{g}(t)^{[2]} \rightarrow 0$ exponentially in the mean square sense. Therefore,

$$
\lim _{t \rightarrow \infty} \tilde{g}(t)=\left[\tilde{g}^{[1]}(0)^{T} \quad \vdots \quad 0 \quad \cdots \quad 0\right] .
$$

As $\hat{g}(t)=\mathrm{T}_{1} \tilde{g}(t)$, it follows that

$$
\lim _{t \rightarrow \infty} \hat{g}(t)=\mathrm{T}_{1}\left[\tilde{g}^{[1]}(0)^{T} \quad: \quad 0 \quad \cdots \quad 0\right]=\mathbf{1} \pi^{\prime} \hat{g}(0)
$$

where $\pi^{\prime}$ is the left eigenvector of the matrix $\alpha^{2} \Gamma$ corresponding to the zero eigenvalue (as in the proof of Theorem 1).

General statements related to the corrected offset estimation still hold in the case of corrected gain estimation. However, the matrix $\alpha^{2} \Gamma$ is now of crucial importance, since its left eigenvector is included in the consensus condition for $\hat{g}(t)$. An unfavorable circumstance in this case could be that small values for $\alpha_{i}$ could have big influence on the $i$-th sensor, which would contradict to the general logic concerning the asymptotic values to be realized in consensus.

\section{Simultaneous Estimation of the GAin AND OfFSET CORReCtion Parameters}

Discussion in Sections 3 and 4 are related to corrected offsets and gains taken individually. Estimation of the corrected offset in Section 3 assumes the gain equal to one. In the general case, the corrected output of the $i$-th sensor, is

$$
\hat{z}_{i}(t)=y_{i}(t)+\hat{b}_{i}(t)=\alpha_{i} x(t)+\beta_{i}+\hat{b}_{i}(t)
$$

and the recursive gradient relation becomes

$$
\hat{f}_{i}(t+1)=\hat{f}_{i}(t)+\delta_{i}(t) \sum_{j \in N_{i}} \gamma_{i j}\left[\hat{f}_{j}(t)-\hat{f}_{i}(t)+\left(\alpha_{j}-\alpha_{i}\right) x(t)\right] .
$$

It is obvious that the element $\left(\alpha_{j}-\alpha_{i}\right) x(t)$ allows neither asymptotic consensus nor convergence of $\hat{f}_{i}(t)$ to possibly different values. This is a consequence of the stochastic nature of signal $x(t)$, the mean value of which is not equal to zero in the general case. However, if we adopt 


$$
\hat{z}_{i}(t)=\hat{a}_{i}(t) y_{i}(t)+\hat{b}_{i}(t)=\hat{a}_{i}(t) \alpha_{i} x(t)+\hat{a}_{i}(t) \beta_{i}+\hat{b}_{i}(t)
$$

the error $\varepsilon_{i j}(t)=\hat{z}_{j}(t)-\hat{z}_{i}(t)$ becomes

$$
\varepsilon_{i j}(t)=\left(\hat{a}_{j}(t) \alpha_{j}-\hat{a}_{i}(t) \alpha_{i}\right) x(t)+\hat{a}_{j}(t) \beta_{j}-\hat{a}_{i}(t) \beta_{i}+\hat{b}_{j}(t)-\hat{b}_{i}(t) .
$$

In relations (44) and (45) $\hat{a}_{j}(t)$ and $\hat{a}_{i}(t)$ are arbitrary estimates of the calibration function parameters. If the estimates $\hat{a}_{j}(t)$ and $\hat{a}_{i}(t)$ had been generated by the algorithm presented in Section 4 , then the asymptotic consensus would have been achieved, implying $\varepsilon_{i j}(t)=0$ in the offset estimation process, having in mind that $\hat{b}_{j}(t)+\hat{a}_{j}(t) \beta_{j}=\hat{b}_{i}(t)+\hat{a}_{i}(t) \beta_{i}$, i.e., all the corrected offsets would have been equal. The idea of estimating parameters $\hat{a}_{i}$ and $\hat{b}_{i}$ simultaneously follows from this observation, in a such way that:

1) The algorithm for gain estimation generates $\hat{a}_{i}(t)$ according to the procedure (26), independently of the offset values;

2) The algorithm for offset estimation generates $\hat{b}_{i}(t)$ according to procedure (9), in which $\varepsilon_{i j}(t)$ is given (45).

The complete calibration algorithm can be represented now as

$$
\begin{gathered}
\hat{a}_{i}(t+1)=\hat{a}_{i}(t)+\delta_{i}(t) \sum_{j \in N_{i}} \gamma_{i j} \varepsilon_{i j}^{\Delta}(t) \Delta y_{i}(t) \\
\hat{b}_{i}(t+1)=\hat{b}_{i}(t)+\delta_{i}(t) \sum_{j \in N_{i}} \gamma_{i j} \varepsilon_{i j}(t)
\end{gathered}
$$

where $\varepsilon_{i j}(t)=\hat{z}_{j}(t)-\hat{z}_{i}(t)$, and $\hat{z}_{i}(t)=\hat{a}_{i}(t) y_{i}(t)+\hat{b}_{i}(t)$. The algorithm (46) and (47) implies communication of $\Delta z_{i}(t)$ and $\hat{z}_{i}(t)$ between the nodes, as well as generation of local corrected outputs by using current values of the parameters $\hat{a}_{i}(t)$ and $\hat{b}_{i}(t)$. The nature of recursions (46) and (47) can be better perceived using the form in which $\hat{a}_{i}(t)$ and $\hat{b}_{i}(t)$ are replaced by the corrected gain $\hat{g}_{i}(t)=\hat{a}_{i}(t) \alpha_{i}$ and corrected offset $\hat{f}_{i}(t)=\hat{a}_{i}(t) \beta_{i}+\hat{b}_{i}(t)$. From (46) and (47) we have

$$
\begin{gathered}
\hat{g}_{i}(t+1)=\hat{g}_{i}(t)+\delta_{i}(t) \sum_{j \in N_{i}} \gamma_{i j}\left(\hat{g}_{j}(t)-\hat{g}_{i}(t)\right) \alpha_{i}^{2} \Delta x(t)^{2} \\
\hat{f}_{i}(t+1)=\hat{f}_{i}(t)+\delta_{i}(t)\left[\alpha_{i} \beta_{i} \Delta x(t)^{2}+x(t)\right] \sum_{j \in N_{i}} \gamma_{i j}\left(\hat{g}_{j}(t)-\hat{g}_{i}(t)\right)+ \\
\delta_{i}(t) \sum_{j \in N_{i}} \gamma_{i j}\left(\hat{f}_{j}(t)-\hat{f}_{i}(t)\right)
\end{gathered}
$$

If we define $\hat{\rho}_{i}(t)=\left[\hat{g}_{i}(t) \hat{f}_{i}(t)\right]^{T}$ we get

where

$$
\hat{\rho}_{i}(t+1)=\hat{\rho}_{i}(t)+\delta_{i}(t) \sum_{j \in N_{i}} \gamma_{i j} M_{i}(t)\left(\hat{\rho}_{j}(t)-\hat{\rho}_{i}(t)\right)
$$

$$
M_{i}(t)=\left[\begin{array}{cc}
\alpha_{i}^{2} \Delta x(t)^{2} & 0 \\
\alpha_{i} \beta_{i} \Delta x(t)^{2}+x(t) & 1
\end{array}\right] .
$$

If we adopt $\hat{g}(t)=\left[\begin{array}{lll}\hat{g}_{1}(t) & \cdots & \hat{g}_{n}(t)\end{array}\right]^{T}$ and $\hat{f}(t)=\left[\begin{array}{llll}\hat{f}_{1}(t) & \cdots & \hat{f}_{n}(t)\end{array}\right]^{T}$, we get the compact forms of the described algorithms

$$
\begin{gathered}
\hat{g}(t+1)=\left(I+\Delta(t) \alpha^{2} \Gamma \Delta x(t)^{2}\right) \hat{g}(t) \\
\hat{f}(t+1)=(I+\Delta(t) \Gamma) \hat{f}(t)+\Delta(t) N(t) \Gamma \hat{g}(t)
\end{gathered}
$$


where $N(t)=\operatorname{diag}\left\{\alpha_{1} \beta_{1} \Delta x(t)^{2}+x(t), \ldots, \alpha_{n} \beta_{n} \Delta x(t)^{2}+x(t)\right\}$. In a similar way, for $\hat{\rho}(t)=\left[\begin{array}{lll}\hat{\rho}_{1}(t)^{T} & \cdots & \hat{\rho}_{n}(t)^{T}\end{array}\right]^{T}$, we get

$$
\left.\hat{\rho}_{i}(t+1)=\left(I+M(t) \Delta(t) \Gamma \otimes \mathrm{I}_{2}\right)\right) \hat{\rho}(t)
$$

where $M(t)=\operatorname{diag}\left\{M_{1}(t), \ldots, M_{n}(t)\right\}$, while $\otimes$ denotes the Kronecker matrix product [23].

We start the convergence analysis from (52) and (53), where the corrected gain and offset vectors are separated. It is clear that all the conclusions from Section 4 are valid for $\hat{g}(t)$. It remains to analyze the asymptotic behavior of the algorithm for $\hat{f}(t)$. It is clear that the conclusions regarding offset estimation from Section 3 are here inapplicable, due to a different definition of the equivalent offset and the existence of an additional element in $\hat{f}(t)$ which depends on $\hat{g}(t)$. This element is stochastic and depends on both $x(t)$ and $\Delta x(t)$.

Theorem 3: Let the Assumptions A), B) and C) be satisfied. Then, the algorithm (50) tends to consensus in the mean square sense, i.e. $\lim _{t \rightarrow \infty} E\left\{\left\|\hat{g}(t)-m_{1} \mathbf{1}\right\|^{2}\right\}=0$ and $\lim _{t \rightarrow \infty} E\left\{\left\|\hat{f}(t)-m_{2} \mathbf{1}\right\|^{2}\right\}=0$, where $m_{1}$ and $m_{2}$ are random variables.

Proof: Behavior of $\hat{g}(t)$ directly follows from Theorem 2, where $m_{1}=\pi^{\prime} \hat{g}(0)$ and $\pi^{\prime}$ is defined within Theorem 2 . We can see that the convergence law is exponential, i.e., $E\left\{\left\|\hat{g}(t)-m_{1} \mathbf{1}\right\|\right\} \leq k_{1} \rho_{1}^{t}$, where $k_{1}<\infty$ and $0<\rho_{1}<1$.

Properties of the recursion for $\hat{f}(t)$ in (50) will be analyzed by adopting $\tilde{f}(t)=T^{-1} \hat{f}(t)$, where $T$ is the transformation defined in Lemma 1; therefore, we get

$$
T^{-1} \hat{f}(t+1)=T^{-1} \hat{f}(t)+\delta T^{-1} \Gamma T T^{-1} \hat{f}(t)+\delta T^{-1} N(\mathrm{t}) \Gamma T_{1} T_{1}{ }^{-1} \hat{g}(t)
$$

where matrix $T_{1}$ is defined in Theorem 2. Considering the form of matrix $\alpha^{2} \Gamma$ (related to $T_{1}$ ), it is clear that the right eigenvector of this matrix corresponding to the zero eigenvalue is equal to vector $\mathbf{1}$. Since $\Gamma \mathbf{1}=0$, it follows that $\Gamma T_{1}$ is the matrix the first column of which is composed of zeros. This means that

$$
T^{-1} N(\mathrm{t}) \Gamma T_{1}=\left[\begin{array}{ll}
0 & N_{\Gamma}(t)^{[1]} \\
0 & N_{\Gamma}(t)^{[2]}
\end{array}\right]
$$

where $N_{\Gamma}(t)^{[1]}$ is an $(n-1)$-dimensional row vector and $N_{\Gamma}(t)^{[2]}$ an $(n-1) \times(n-1)$ matrix. We can see that $N_{\Gamma}(t)^{[1]}$ and $N_{\Gamma}(t)^{[2]}$ depend on the random variables $\Delta x(t)$ and $x(t)$. Consequently, $\tilde{f}(t)=T^{-1} \hat{f}(t)$ can be decomposed in the following way

$$
\begin{gathered}
\tilde{f}(t+1)^{[1]}=\tilde{f}(t)^{[1]}+\delta N_{\Gamma}(t)^{[1]} \tilde{g}(t)^{[2]} \\
\tilde{f}(t+1)^{[2]}=\left(I+\delta \Gamma^{*}\right) \tilde{f}(t)^{[2]}+\delta N_{\Gamma}(t)^{[2]} \tilde{g}(t)^{[2]}
\end{gathered}
$$

where $\tilde{f}(t)=\left[\tilde{f}_{1}(t)^{[1]} \quad: \quad \tilde{f}(t)^{[2] T}\right]^{T}$ and $\tilde{g}(t)^{[2]}$ is defined in Theorem 2. The first recursion in (57) gives

$$
\lim _{t \rightarrow \infty} \tilde{f}(t)^{[1]}=\tilde{f}(0)^{[1]}+\delta \lim _{t \rightarrow \infty} \sum_{k=0}^{t-1} N_{\Gamma}(k)^{[1]} \tilde{g}(k)^{[2]}=\tilde{f}(0)^{[1]}+m_{2}^{\prime} .
$$

Random variable $m_{2}^{\prime}$ satisfies

$$
E\left\{\left|m_{2}^{\prime}\right|\right\} \leq \delta \lim _{t \rightarrow \infty} \sum_{k=0}^{t-1} E\left\{E\left\{\left\|N_{\Gamma}(k)^{[1]}\right\| \mid F_{k}\right\}\left\|\tilde{g}(k)^{[2]}\right\|\right\} \leq
$$




$$
k_{1}^{\prime} \lim _{t \rightarrow \infty} \sum_{k=0}^{t-1} E\left\{\left\|\widetilde{g}(k)^{[2]}\right\|\right\} \leq k_{2}^{\prime} \lim _{t \rightarrow \infty} \sum_{k=0}^{t-1} \rho_{2}^{k}<k_{3}^{\prime}<\infty
$$

where $k_{1}^{\prime}, k_{2}^{\prime}, k_{3}^{\prime}$ i $0<\rho_{2}<1$ are generic constants. The second recursion in (58) gives

$$
\tilde{f}(t)^{[2]}=\left(I+\delta \Gamma^{*}\right)^{t-1} \tilde{f}(0)^{[2]}+\delta \sum_{k=0}^{t-1}\left(I+\delta \Gamma^{*}\right)^{t-k-1} N_{\Gamma}(t)^{[2]} \tilde{g}(t)^{[2]}
$$

From here it follows that

$$
E\left\{\left\|\tilde{f}(t)^{[2]}\right\|\right\} \leq k_{1}^{\prime \prime} \sum_{k=0}^{t-1} \rho_{1}^{t-k} \rho_{2}^{k} \underset{t \rightarrow \infty}{\longrightarrow} 0
$$

where $k_{1}^{\prime \prime}<\infty, 0<\rho_{1}, \rho_{2}<1$, since $E\left\{\left\|N_{\Gamma}(t)^{[1]}\right\|^{2} \mid F_{k}\right\}$ is bounded and $E\left\{\left\|\tilde{g}(k)^{[2]}\right\|^{2}\right\}$ exponentially tends to zero. The conclusion is that in the mean square sense

$$
\lim _{t \rightarrow \infty} \tilde{f}(t)=T \tilde{f}(0)=T\left[\begin{array}{c}
\tilde{f}(0)+m_{2}^{\prime} \\
\cdots \\
0 \\
\vdots \\
0
\end{array}\right]=\mathbf{1}\left(\pi \hat{f}(0)+m_{2}^{\prime}\right)=\mathbf{1} m_{2}
$$

having in mind the structure of matrices $T$ and $T^{-1}$.

The result of Theorem 3 shows that consensus can be achieved simultaneously for both calibration function parameters. It should be also observed that in this case random variable $m_{2}^{\prime}$ results, at least partially, from the impact of $\hat{g}(t)$ on the recursion for $\hat{f}(t)$. It is important that $\hat{g}(t)$ tends to consensus exponentially.

\section{SimULATION RESULTS}

Basic properties of the proposed algorithms are illustrated by simulations related to a sensor network with ten nodes. Two different cases are taken into consideration:

1) $G_{1}$ - sensor network with randomly chosen weighting coefficient values $\gamma_{i j}$

2) $G_{2}$ - sensor network obtained from $G_{1}$ by removing many of the connections between the nodes, but still satisfying assumption B). In this way we get a graph with a lower degree of connectedness.

The measured signal has been generated by a second order constant parameter ARMA process with steady state variance equal to one, satisfying assumption C)

In Figs. 1-3 corrected offsets generated by proposed algorithm in Section 3 are represented. Fig. 1 is an illustration of time evolution of the corrected offsets for all ten nodes in $G_{1}$ with the step size of the algorithm tuned at 0.01 . The convergence is obviously very fast and it proves the efficiency of the algorithm mentioned above. In Fig. 2 the time evolution of the corrected offsets is shown, but with the step size set to 0.001 . It is clear that in this case the convergence is slower. Fig. 3 shows how the degree of connectedness affects the convergence properties. The time evolution of the corrected offsets is shown for the case $G_{2}$. From the comparison of Fig. 3 with Fig. 1 it clear that the convergence is slower for the sensor network with a lower degree of connectedness. 


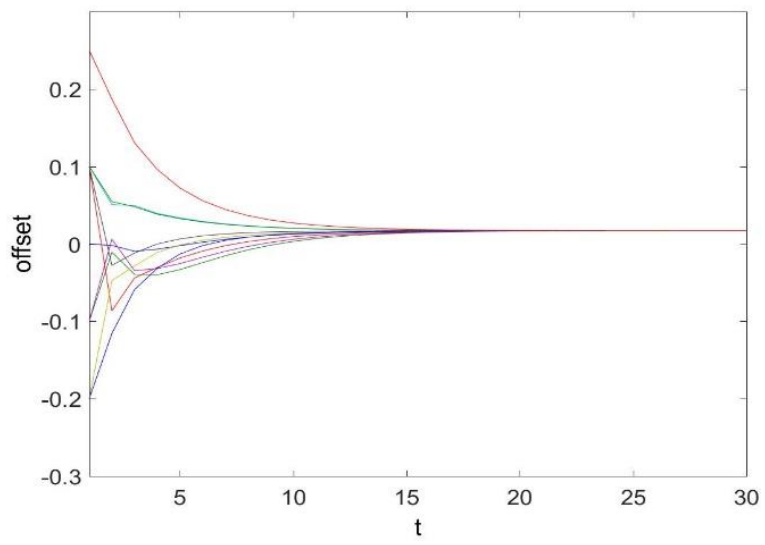

Fig. 1 Time evolution of the corrected offsets, case $G_{1}, \delta_{i}=\delta=0.01$

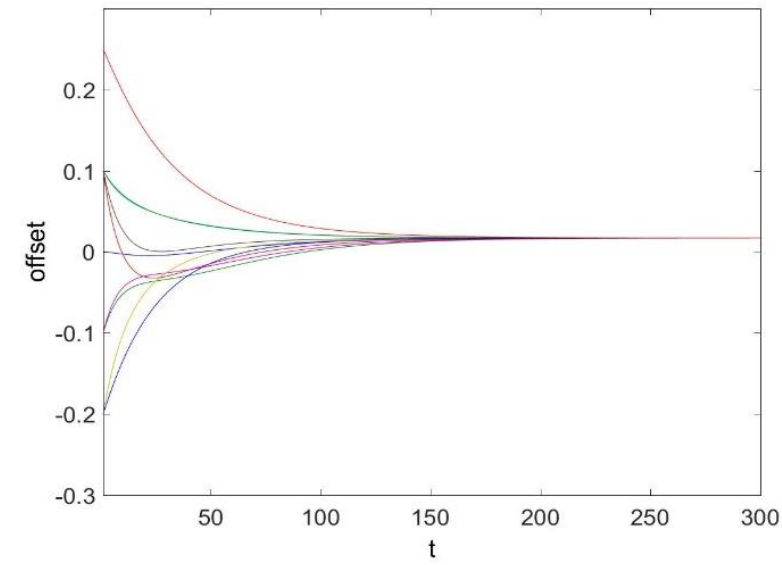

Fig. 2 Time evolution of the corrected offsets, case $G_{1}, \delta_{i}=\delta=0.001$

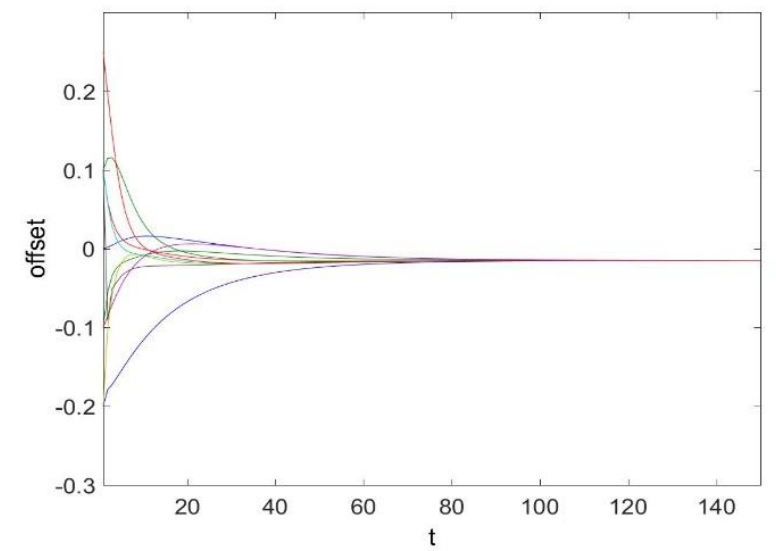

Fig. 3 Time evolution of the corrected offsets, case $G_{2}, \delta_{i}=\delta=0.01$ 
In Figs.4-6 the corrected gains are represented, generated by the algorithm proposed in Section 4. Fig. 4 shows time evolution of the corrected gains for the case $G_{1}$, with the step size set to 0.01 .

Fig. 5 illustrates the influence of the algorithm step size itself. All settings are the same as in the case of described for Fig. 4, except for the step size, which is set to 0.001. It is clear that the convergence is slower for the lower step size. Fig. 6 corresponds to the case $G_{2}$, with the step size tuned at 0.01 . Once again it is shown how the degree of connectedness influences the convergence rate - the lower degree of connectedness, the slower convergence.

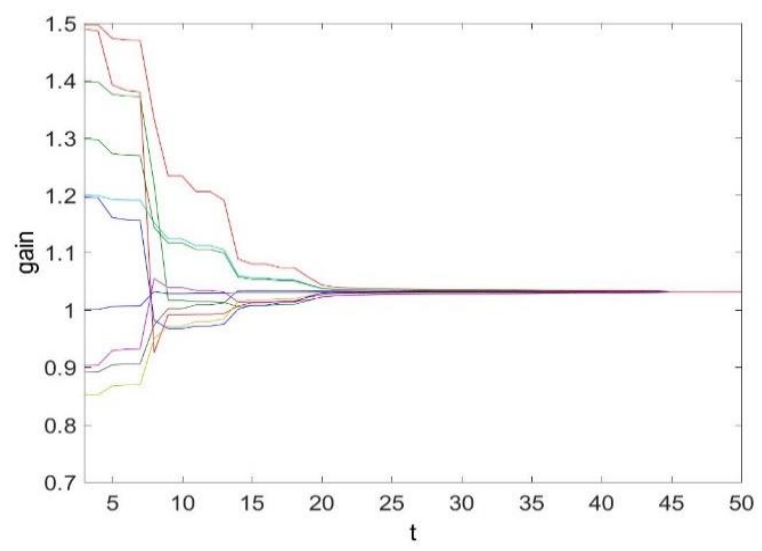

Fig. 4 Time evolution of the corrected gains, case $G_{1}, \delta_{i}=\delta=0.01$

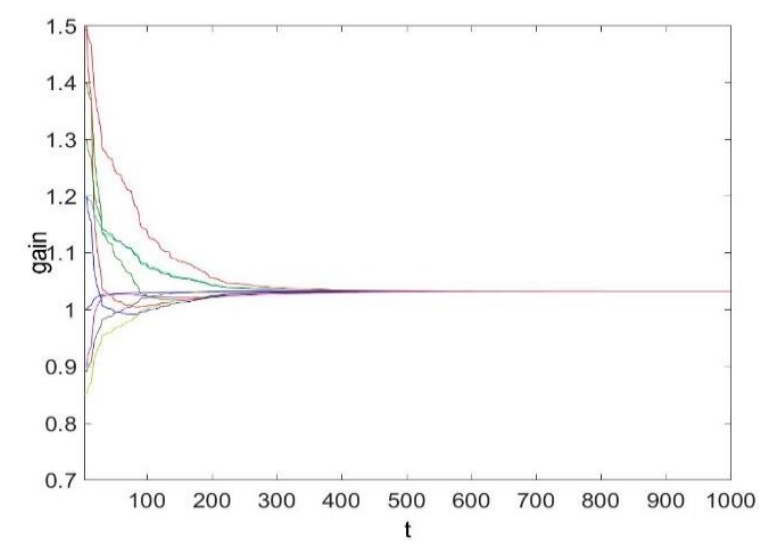

Fig. 5 Time evolution of the corrected gains, case $G_{1}, \delta_{i}=\delta=0.001$ 


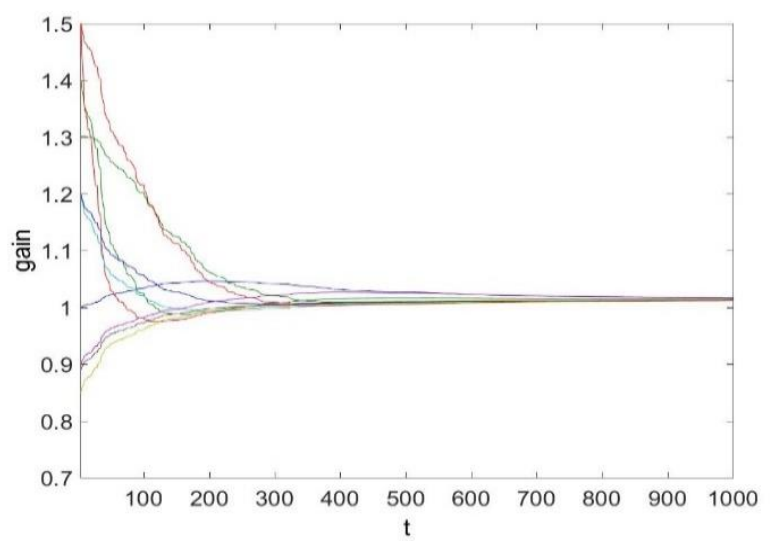

Fig. 6 Time evolution of the corrected gains, case $G_{2}, \delta_{i}=\delta=0.01$

In Figs. 7-9 the simultaneous convergence of the gains and offsets are illustrated. Fig. 7 corresponds to the proposed algorithm, and Fig. 8 to the algorithm proposed in $[7,11]$, both with delta $=0.01$. It is obvious that the proposed algorithm provides a superior convergence rate, as expected. Numerous simulations show that the proposed algorithm can serve as a more efficient tool in practice. In Fig. 9 the correction parameters are represented in the case of delta $=0.0001$, as an additional illustration of the influence of the step size of the algorithm on the overall convergence rate.

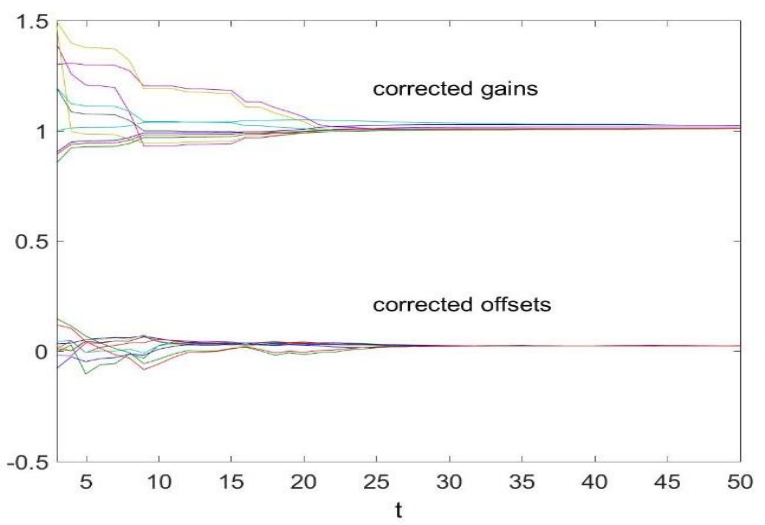

Fig. 7 Time evolution of the corrected parameters for the proposed algorithm with $\delta_{i}=\delta=0.01$ 


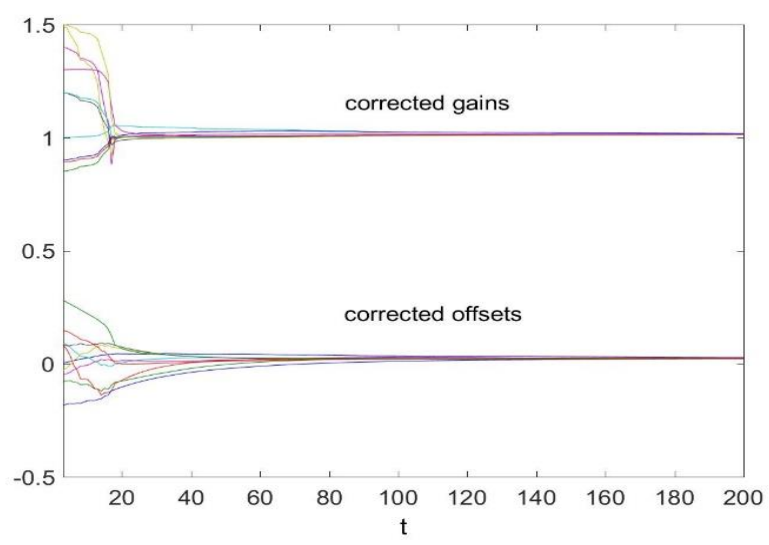

Fig. 8 Time evolution of the corrected parameters for the algorithm proposed in $[7,11]$ with the same gain as in Fig. 7

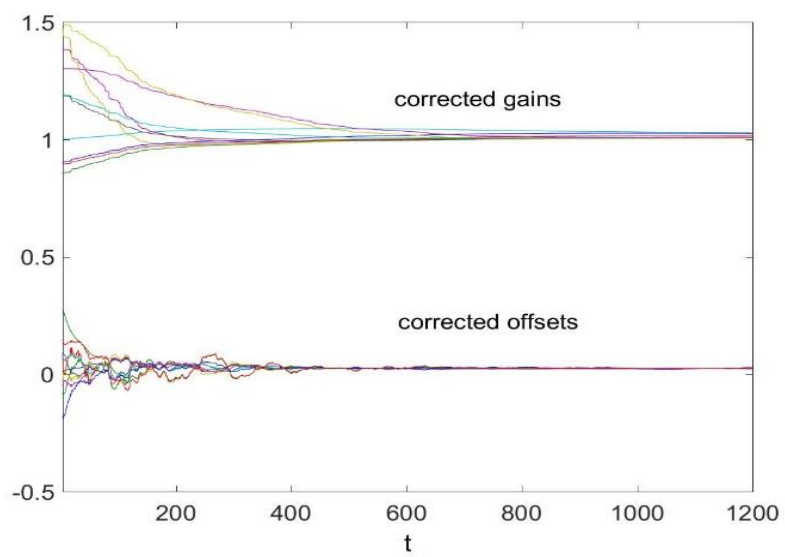

Fig. 9 Time evolution of the corrected parameters for $\delta_{i}=\delta=0.0001$

\section{CONCLUSION}

In this paper a new algorithm for distributed blind macro calibration of sensor networks is presented, consisting of two recursive gradient based algorithms for estimating gain and offset correction parameters of all the sensors in the network. The algorithm does not require the knowledge of the measured signal. The algorithm for gain correction is derived from measured signal increments, and functions independently. The algorithm for offset correction utilizes the gain correction parameters given by the first algorithm and the signal measurement error. It is demonstrated that the entire calibration algorithm can be treated as two dynamic consensus algorithms. It provides asymptotically equal corrected gains and equal corrected offsets for all the sensors in the network.

The paper contains proofs of the convergence of the offset and gain correction parameters taken separately, as well of the whole algorithm based on simultaneous estimation of both 
gain and offset correction parameters. It is demonstrated that the algorithm provides the exponential convergence to consensus. It is also indicated that the proposed algorithm structure offers a possibility to achieve a convergence rate superior to the existing similar schemes. Selected simulation results illustrate the main properties of the proposed algorithm and its advantage over the algorithm proposed in $[7,11]$ from the point of view of the convergence rate.

Further research could be oriented towards robustifying the presented algorithm with respect to the measurement and communication noise containing outliers of high intensity. It would be also interesting to study a possible incorporation of a spatial model of the measured signal.

\section{REFERENCES}

[1] Wang, F.Y., Liu, D., Networked Control Systems : Theory and Applications, 2008, Springer, N.Y.

[2] Hespanha, J. P.; Naghshtabrizi, P.; Xu, Y., "A Survey of Recent Results in Networked Control Systems," Proceedings of the IEEE., 2007, 95, pp. 138-162.

[3] Garavi, H., Kumar, S.P. (Eds.), "Sensor Networks and Applications,"IEEE Proc., 2003, 91, No. 8

[4] Whitehouse K. and Culler D., "Macro-calibration in sensor/actuator networks," Mobile Netw. Appl., 2003, 8, pp. 463-472

[5] L. Balzano and R. Nowak, "Blind calibration," Networked and Embedded Systems Laboratory, UCLA, Tech. Rep. TRUCLA- NESL-200702-01, 2007.

[6] R. Olfati-Saber, A. Fax, and R. Murray, "Consensus and cooperation in networked multi-agent systems," in Proc. of the IEEE, vol. 95, pp. 215-233, 2007.

[7] M. S. Stanković, S. S. Stanković, and K. H. Johansson, "Distributed macro calibration in sensor networks," in Proc. 20th Mediterranean Conference on Control and Automation, 2012.

[8] L. Balzano and R. Nowak, "Blind calibration in sensor networks," in Proc. Intern. Conf. Inf. Proc. in Sensor Networks, April 2007.

[9] M. Takruri, S. Challa, and R. Yunis, "Data fusion techniques for auto calibration in wireless sensor networks, “ in Proc. Int. Conf. on Information Fusion, 2009, 1, pp. 132-139

[10] Bychkovsky, V., Megerian, S., Estrin, D. and Potkonjak, M., "A collaborative approach to in-place sensor calibration," Proc. Intern. Conf. Inf. Proc. in Sensor Netw., 2003, pp. 301-316

[11] Stanković, M.S., Stanković, S.S. and Johansson, K.H., "Distributed blind calibration in lossy sensor networks via output synchronization,” IEEE Trans. Autom. Control, 2015, 60, pp. 3257-3262

[12] M. S. Stanković, S. S. Stanković, and K. H. Johansson, "Distributed calibration for sensor networks under communication errors and measurement noise," in Proc. IEEE Conf. Decision and Control, 2012.

[13] P. Sommer and R. Wattenhofer, "Gradient clock synchronization in wireless sensor networks," in Proc. Int. Conf. Inf. Proc. in Sensor Networks, 2009, pp. 37-48.

[14] L. Schenato and F. Fiorentin, "Average timesynch: a consensus-based protocol for time synchronization in wireless sensor networks," Automatica, vol. 47, no. 9, pp. 1878-1886, 2011.

[15] C. Liao and P. Barooah, "Distributed clock skew and offset estimation from relative measurements in mobile networks with Markovian switching topologies," Automatica, vol. 49, pp. 3015-3022, 2013.

[16] Tian, Y.-P., Zong, S., Cao, Q., ,Structural modeling and convergence analysis of consensus-based time synchronization algorithms over networks: nontopological conditions“, Automatica, 2016, 65, pp. 64-75

[17] Stanković, M.S., Stankovic, S.S., Johansson, K.H., "Distributed time synchronization for networks with random delays and measurement noise," Automatica, 2018, 93, pp. 126-137.

[18] Stanković, M.S., Stanković, S.S., Johansson, K.H., “Asynchronous distributed blind calibration of sensor networks under noisy measurements," IEEE Trans.Contr. Netw. Systems, 2018, 5, pp. 571-582

[19] C. Godsil and G. Royle, Algebraic Graph Theory, New York: Springer Verlag, 2001.

[20] W. Ren and R. Beard, "Consensus seeking in multi-agent systems using dynamically changing interaction topologies,” IEEE Trans. Autom. Control, 50:655_661, 2005.

[21] W. Ren, R. W. Beard, and D. B. Kingston, "Multi-agent Kalman consensus with relative uncertainty," in Proc. American Control Conference, 2005.

[22] R. Olfati-Saber, "Distributed Kalman filtering for sensor networks," in Proc. IEEE Conf. Decision and Control, pages 5492_5498, 2007.

[23] Horn, R.A., Johnson, C.R., Matrix Analysis, 2013, Cambridge Univ. Press, Cambridge, U.K. 